\title{
Kinetic Disordering of Intermetallic Compounds Through First- and Second-Order Transitions by Rapid Solidification
}

\section{Citation}

West, Jeffrey A., and Michael J. Aziz. 1992. "Kinetic Disordering of Intermetallic Compounds Through First- and Second-Order Transitions by Rapid Solidification." In Ordering and Disordering in Alloys, ed. A. R. Yavari (Proceedings of the European Workshop on Ordering and Disordering held in Grenoble, France, 10th-12th July, 1991), 23-30. New York: Springer Netherlands. doi:10.1007/978-94-011-2886-5_3.

\section{Published Version}

doi:10.1007/978-94-011-2886-5_3

\section{Permanent link}

http://nrs.harvard.edu/urn-3:HUL.InstRepos:34253798

\section{Terms of Use}

This article was downloaded from Harvard University's DASH repository, and is made available under the terms and conditions applicable to Other Posted Material, as set forth at http:// nrs.harvard.edu/urn-3:HUL.InstRepos:dash.current.terms-of-use\#LAA

\section{Share Your Story}

The Harvard community has made this article openly available.

Please share how this access benefits you. Submit a story.

Accessibility 


\title{
KINETIC DISORDERING OF INTERMETALLIC COMPOUNDS THROUGH FIRST- AND SECOND-ORDER TRANSITIONS BY RAPID SOLIDIFICATION
}

\author{
JEFFREY A. WEST AND MICHAEL J. AZIZ \\ Division of Applied Sciences \\ Harvard University \\ Cambridge, MA 02138, USA
}

\begin{abstract}
During rapid solidification of intermetallic compounds, the atoms may not have time to find the lowest-energy sites in the crystal, resulting in the growth of a solid with partially or completely suppressed chemical order. A kinetic model has been developed for "disorder trapping" during rapid solidification, which predicts the long range order parameter, composition and temperature at the interface of a chemically ordered phase as functions of interface velocity and liquid composition. The model predicts that as the solidification velocity increases, the long range order parameter decreases. Beyond a critical velocity the order parameter is zero. The predicted transition to solidification of a disordered solid is discontinuous for thermodynamically first-order cases, and continuous for thermodynamically second-order cases.

$\mathrm{Ni}_{2} \mathrm{TiAl}\left(\mathrm{L}_{2}\right)$ and $\mathrm{Ni} 3 \mathrm{Al}\left(\mathrm{L}_{2}\right)$, which in equilibrium are ordered to their melting points, have been kinetically disordered by rapid solidification following pulsed laser melting. The order-disorder transitions are second-order and first-order, respectively. The interface velocity and order parameter can be followed with nanosecond resolution by monitoring the reflectivity and lateral resistance of a thin film sample during and immediately after solidification. X-ray diffraction and Transmission Electron Microscopy (TEM) indicate that metastable fcc $\mathrm{Ni}_{3} \mathrm{Al}$ was retained by quenching. In rapidly solidified bulk Ni2 TiAl TEM reveals a fine array of antiphase boundaries, indicating that the material formed from the melt with the unstable B2 structure and subsequently transformed to the equilibrium Heusler structure during cooling to room temperature. In rapidly solidified $\mathrm{Ni}_{2} \mathrm{TiAl}$ thin films, electron diffraction indicates that the unstable bcc structure was formed directly from the melt and retained upon cooling.
\end{abstract}

\section{INTRODUCTION}

Intermetallic compounds are attractive prospects for use as high-temperature, high-strength, corrosion-resistant materials. Their lack of room-temperature ductility, however, can prevent their widespread use by limiting their formability. Many of these materials might become more practical if they could be put into a metastable chemically disordered phase ("disorder 
trapping") that was less difficult to machine or forge at room temperature to near net shape. Subsequently, the part could be annealed to bring out the chemical order and the associated high-temperature properties. Furthermore, some line compounds might become useful if they could be formed off stoichiometry ("solute trapping"). In addition to the known improvement in ductility that sometimes accompanies deviations from stoichiometry, such materials may become useful intermediate states in the production of practical alloys. Phases of nonequilibrium composition might serve as ideal starting materials for the use of subsequent thermomechanical processing to produce an extremely fine dispersion of precipitates that resist coarsening and re-dissolution at high temperatures, thus pinning grain boundaries and providing superior high-temperature strength. In addition to their potential technological relevance, kinetically disordered materials may serve as ideal starting points for studies of ordering kinetics, or for studies of the effect of ordering on properties.

These possibilities have been investigated by studying theoretically and experimentally the chemical long-range order (LRO) of rapidly solidified intermetallic compounds, and by studying theoretically the expected deviations from stoichiometry that could be induced by rapid solidification. This paper summarizes this work. Boettinger and Aziz developed a model for "disorder trapping" [1]. Boettinger, Bendersky, Cline, West, and Aziz formed the unstable B2 phase in bulk specimens of $\mathrm{Ni}_{2} \mathrm{TiAl}$ [2]. West, Manos, and Aziz formed the metastable fcc phase of $\mathrm{Ni}_{3} \mathrm{Al}$ [3] and the unstable bcc phase of $\mathrm{Ni}_{2} \mathrm{TiAl}_{3}[4]$ in thin film samples.

\section{MODEL}

The Boettinger-Aziz model for solute and disorder trapping by rapid solidification predicts the deviation from stoichiometry and the deviation from the equilibrium LRO parameter, as functions of the melt composition and the velocity of the crystal/melt interface during rapid solidification. The model predicts that:

(1) Compounds that are normally ordered to the melting point will solidify with trapped anti-site disorder at high solidification velocities. With increasing interface velocity the LRO parameter, $\eta$, will decrease. For transitions that are thermodynamically second order, $\eta$ decreases continuously, reaching zero at a critical velocity as shown in Fig. 1(a). For orderdisorder transitions that are thermodynamically first order, $\eta$ first decreases from the equilibrium value and then makes a discontinuous jump to zero at a critical velocity as shown in Fig. 1(b). A simple analytic expression was developed for $v_{\mathrm{T}}$, the critical velocity for formation of a completely disordered alloy, in both cases. For the second-order case, characteristic of the B2 $\rightarrow$ bcc transition, the critical velocity is given by a very simple expression for a stoichiometric Bragg-Williams binary alloy: 


$$
v_{\mathrm{T}}=v_{\mathrm{D}}\left[\left(\mathrm{T}_{\mathrm{C}} / \mathrm{T}_{\mathrm{M}}\right)-1\right] \text {, }
$$

where $v_{D}$ is the ratio of the interface diffusivity to the jump distance, $T_{C}$ is the critical temperature for the order-disorder transition and $\mathrm{T}_{M}$ is the solidus temperature of the compound. Only phases that are ordered up to their solidus point are considered; i.e., $\mathrm{T}_{C} / \mathrm{T}_{M}$ $>1$. Large values of $T_{C}$, which relate directly to the strength of ordering, require large velocities to trap complete disorder.
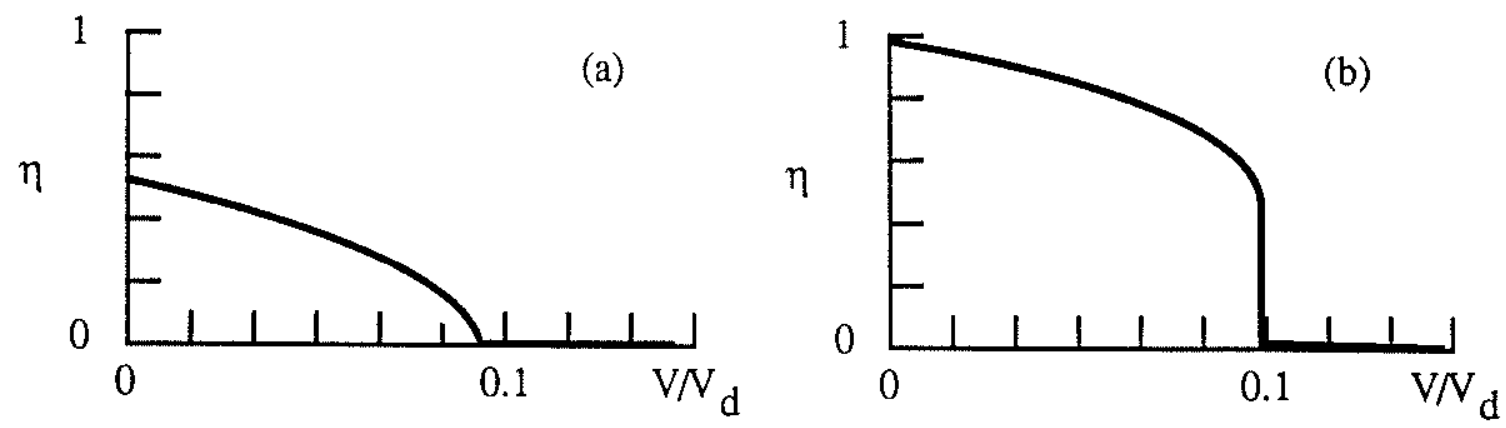

Figure 1. Predicted long range order parameter of the growing solid at the interface, $\eta$, as a function of dimensionless growth velocity, $v / v_{D}$, for a congruently melting stoichiometric $A B$ compound. Predictions are made for cases where order-disorder transition is thermodynamically (a) second order; (b) first order.

(2) Deviations from stoichiometry can be trapped in by rapid solidification. For solidification from an off-stoichiometric melt, the solid's deviations from stoichiometry increase with increasing solidification velocity. The details depend on the free energy of the solid as a function of composition and order parameter, which is not yet known for most compounds but could, in many cases, be calculated by the cluster variation method [5].

(3) The solid/liquid interface is highly mobile during the solidification of completely disordered or completely ordered compounds, but is much more sluggish at intermediate velocities where partial disorder trapping or partial solute trapping is occurring. This implies the possibility of a competitive growth situation in which a metastable disordered phase nucleates and out-races a growing, partially-ordered phase.

\section{EXPERIMENTAL TECHNIQUE}

\section{Sample preparation}

Thin film samples. Thin films on insulators are used to facilitate the transient electrical resistance measurement of the interface velocity while avoiding electrical short-circuiting from the substrate. In order to ensure plane-front melting and subsequent solidification (necessary for a quantitative measurement of the interface velocity) it is essential that we have starting materials with surfaces that are smooth to a few hundred $\AA$, providing uniform absorption of 
laser irradiation and uniform heat flow into the substrate. Two substrates that we have used successfully are sapphire and thermally oxidized Si wafers. Thin films are prepared by vacuum evaporation or by ion beam sputtering, using a multiple-target sputtering system or a dual-hearth e-beam evaporator. Thin film samples are photolithographically patterned into a geometry for optimizing signal-to-noise during ultrafast electrical measurements.

Bulk Specimens. Initial experiments on $\mathrm{Ni}_{2} \mathrm{TiAI}$ were done on bulk specimens. They were prepared by arc melting, diamond sawing and spark cutting.

\section{Time-resolved measurements}

Transient conductance and optical reflectance. Melting and solidification induced by pulsed laser melting of semiconductors can easily be monitored with a time resolution of a few nanoseconds. Large optical reflectivity changes indicate when melting or resolidification occur in the first few nanometers adjacent to the surface. By measuring the transverse electrical conductance of a thin film sample whose melt has a different resistivity than does the crystal, as shown in Fig. 2, the melt depth and interface velocity can be measured [6] over depths greater than half a micron and with time resolution of down to one nanosecond, depending upon the bandwidth of one's oscilloscope. The changes in electrical conductivity and optical reflectance upon melting of many elemental metals and alloys are sufficiently large to allow the same transient electrical conductance measurement of melt depths and velocities as has been used
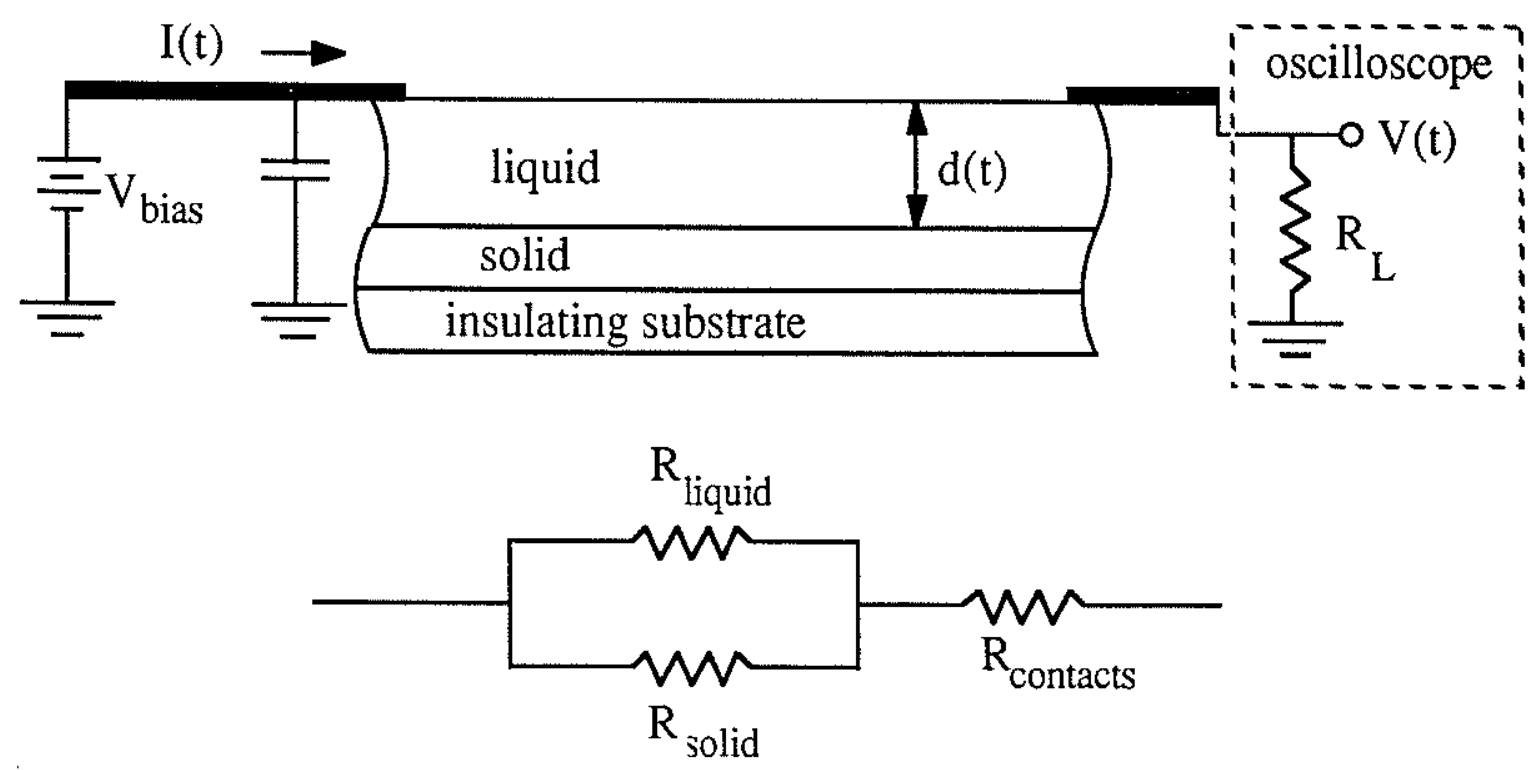

Figure 2. Transient conductance measurement and equivalent DC circuit.

successfully on semiconductors. We have made these measurements on several elemental metallic thin films $[7,8]$, as well as on intermetallic compounds $[3,4]$. The accuracy is not as 
great as for $\mathrm{Si}$, but any measurement at all of the interface velocity is a vast improvement over the alternative, which is a mere estimate of the growth rate from heat flow calculations.

Pulsed Laser Melting. Laser irradiation is performed with an ultraviolet (308 nm) pulsed excimer laser. The laser pulse is rendered spatially uniform by passing it through an external beam homogenizer. The pulse is roughly 30 nanoseconds in duration, routinely yielding solidification rates of a few $\mathrm{m} / \mathrm{s}$ in metal films on $\mathrm{SiO}_{2}$ substrates.

\section{Post-irradiation analysis}

We have used plan-view transmission electron microscopy (TEM) to examine the structure and crystallinity of laser irradiated material, on a Philips 420 TEM-STEM. TEM is usually sufficient to detect superlattice reflections and characteristics of the microstructure such as grain size, anti-phase boundary configuration, and precipitation. Rutherford Backscattering Spectrometry is used to obtain film thicknesses and compositions. Quantitative measurements of the LRO parameter are made with X-ray diffraction, performed on a GE $\theta-2 \theta$ diffractometer using $\mathrm{Cr} K_{\alpha}$ radiation. In the $\theta-2 \theta$ geometry, only crystals having atomic planes parallel to the film surface contribute to diffraction. Monitoring a superlattice and a fundamental reflection pair with a scattering direction in common (e.g., 110 and 220) guarantees that the same crystals are responsible for diffraction into both peaks, eliminating any problem arising from possible texturing during any of the processing steps.

\section{RESULTS}

\section{Disorder trapping in $\mathrm{Ni}_{3} \mathrm{Al}$}

$\mathrm{Ni} 3 \mathrm{Al}$ is ordered to its melting point, above which lies a metastable order-disorder transition from $\mathrm{Ll}_{2}$ to fcc that is thermodynamically first-order. Its disorder-trapping behavior is predicted to behave as in Fig. 1(b). It is found to form a completely disordered fcc phase upon rapid solidification at velocities of 2 meters per second and above, and to re-order into the $\mathrm{L}_{2}$ phase during subsequent furnace annealing. The LRO was monitored by an X-ray diffraction measurement of the ratio of the intensity of the 110 superlattice peak to that of the 220 fundamental peak in $\theta-2 \theta$ geometry. The 110 superlattice peak completely disappeared following rapid solidification, and returned after furnace annealing. This result confirms the prediction of the model that complete disorder trapping is possible by rapid solidification.

\section{Disorder trapping in $\mathrm{Ni}_{2} \mathrm{TiAl}$}

The Heusler alloy $\mathrm{Ni}_{2} \mathrm{TiAl}$, with the $\mathrm{L}_{2}{ }_{1}\left(\mathrm{DO}_{3}\right)$ structure, can have two levels of disorder, characterized by two order parameters. If the order parameter for the Ti-Al sublattice 
goes to zero, then $\mathrm{Ti}$ and $\mathrm{Al}$ randomly populate this sublattice and the structure becomes $\mathrm{B} 2$; if the other order parameter subsequently vanishes it becomes bcc. Each of these transitions has a unique $\mathrm{T}_{\mathrm{C}}$, so the application of equation (1) indicates that as the solidification velocity is increased, first $\mathrm{L} 21$, then $\mathrm{B} 2$, then bcc will form from the melt.

We have laser-melted bulk specimens of $\mathrm{Ni}_{2} \mathrm{TiAl}$. The melt duration was measured and used to calibrate heat-flow calculations, which estimated the velocity during subsequent solidification to be approximately $8-20 \mathrm{~m} / \mathrm{s}$. TEM analysis of the solidified material revealed the $\mathrm{L} 21$ phase with a fine dispersion of anti-phase boundaries. The boundaries all had $<100>$ displacements, indicating that they result from a B2 $\rightarrow \mathrm{L} 21_{1}$ transition. Since no boundaries were found with $<111>$ displacements, which would be characteristic of the bcc $\rightarrow$ B2 transition, we inferred that the B2 phase was formed directly from the melt and that it ordered to the equilibrium L2 1 structure during subsequent cooling of the solid from the melting point to room temperature.

Rapid solidification of thin films of $\mathrm{Ni}_{2} \mathrm{TiAl}$ on $\mathrm{SiO}_{2}$ at velocities measured to be $\sim 4 \mathrm{~m} / \mathrm{s}$ yielded altogether different results. Only bcc fundamental reflections are always visible in TEM diffraction patterns, B2 superlattice reflections are missing from some grains, and $L 2_{1}$ superlattice reflections are always absent. Furnace annealing recovers the B2 and L 21 reflections in all cases. This result indicates that completely disordered bcc $\mathrm{Ni}_{2} \mathrm{TiAl}$ can be solidified directly from the melt and retained during cooling to room temperature.

\section{DISCUSSION}

These experiments reviewed here demonstrate that intermetallic compounds can be completely disordered by rapid solidificaton. Yavari and Bochu had formed partially disordered $\mathrm{Ni}_{3} \mathrm{Al}$ by melt spinning [9], but were only able to form completely disordered compounds by adding enough $\mathrm{Fe}$ to depress the critical temperature below the solidus. In the latter case, rather than forming a metastable material directly from the melt, they were forming an equilibrium disordered solid solution from the melt and suppressing solid-state ordering during rapid solidphase cooling. Quantitative estimates of the critical velocity for complete disorder trapping depend in detail on the thermodynamics of $\mathrm{Ni} 3 \mathrm{Al}$ and on the magnitude of $v_{\mathrm{D}}$, the "diffusive speed". For a range of thermodynamic parameters tinkered with while exploring the model, the critical velocity seems to range from near $v_{\mathrm{D}}$ to velocities well below $v_{\mathrm{D}}$, the latter being the case when the driving force for ordering is very slight. Preliminary experiments on solute trapping in disordered metallic alloys [8] yield values of $v_{D}$ on the order of $1 \mathrm{~m} / \mathrm{s}$, which lies in the realm of pulsed laser melting. As solidification and cooling rates from melt spinning are several orders of magnitude less than those due to pulsed laser melting, complete disorder trapping by rapid solidification following pulsed laser melting and partial disorder trapping by 
melt spinning seem to be near extreme cases for the range of possibilities. A more complete and quantitative test of the model, by measuring the LRO parameter over a range of velocities, has yet to be performed.

The formation of unstable disordered $\mathrm{Ni}_{2} \mathrm{TiAl}$ by suppressing second order transitions during rapid solidification may come as a surprise to some. To form such a material, one must score a kinetic "bull's eye"; i.e., forming a phase with a configuration close to the target state is insufficient, because relaxation will occur in a direction away from the target state. Apparently, the kinetics of rapid solidification lead directly to that bull's eye. The solidification and cooling rate must be so high that virtually no time is available for the barrierless relaxation to an ordered structure. The necessary solidification velocity in this second-order case is estimated using eq. (1). Burton et al. [10] have estimated the critical temperatures for the $\mathrm{L} 2 \mathrm{~L}_{1} \rightarrow \mathrm{B} 2$ and the B2 $\rightarrow$ bcc transitions as $1900 \mathrm{~K}$ and $3300 \mathrm{~K}$, respectively. With a melting point of about $1800 \mathrm{~K}$ for ordered $\mathrm{Ni} 2 \mathrm{TiAl}$ and a $v_{\mathrm{D}}$ of $1 \mathrm{~m} / \mathrm{s}$, eq. (1) predicts B2 formation above $0.6 \mathrm{~m} / \mathrm{s}$ and bcc formation above $9 \mathrm{~m} / \mathrm{s}$. This is roughly consistent with the experimental velocities. We had expected that solidification of thin films on $\mathrm{SiO}_{2}$ would occur more slowly than on bulk samples, resulting in a more highly ordered solid. However, it is possible that the thin film samples undercooled significantly prior to solidification in the absence of a crystal seed, and then grew very rapidly once nucleation occurred. There might have been some amount of lateral growth of crystalline nuclei, this would prevent us from interpreting the transient conductance measurement in terms of an interface velocity. Extreme undercoolings from pulsed laser melting are not uncommon; we have hypercooled liquid $\mathrm{Ni}(\Delta \mathrm{T} \approx 512 \mathrm{~K})$ by this method [7].

\section{CONCLUSIONS}

Rapid solidification forms metastable compositionally-disordered fcc $\mathrm{Ni} 3 \mathrm{Al}$ and unstable compositionally-disordered $\mathrm{B} 2$ and bcc Ni2 $\mathrm{TiAl}$, as predicted by the model for disorder trapping. The observed velocities for solidification of disordered alloys are roughly consistent with theoretically estimated critical solidification velocities for complete disorder trapping.

\section{ACKNOWLEDGMENTS}

Some of the work reported here is the result of a collaboration with W.J. Boettinger, L.A. Bendersky, and J. Cline at NIST. We are grateful to J.T. Manos and P.M. Smith at Harvard for technical assistance. Work at Harvard has been supported by N00014-88-K-0548. 


\section{REFERENCES}

[1] W.J. Boettinger and M.J. Aziz, Acta Metall. 37, 3379 (1989).

[2] W.J. Boettinger, L.A. Bendersky, J.A. West, M.J. Aziz and J. Cline, Mat. Sci. Eng. A133, 592 (1991).

[3] J.A. West, J.T. Manos, M.J. Aziz, Mat. Res. Soc. Symp. Proc. 213, (in press, 1991).

[4] J.A. West, Ph.D. thesis, Harvard University, in preparation (1991).

[5] B.P. Burton, J.E. Osburn, and A. Pasturel, Mat. Res. Soc. Symp. Proc. 213, (in press, 1991).

[6] M.O. Thompson, J.W. Mayer, A.G. Cullis, H.C. Webber, N.G. Chew, J.M. Poate and D.C. Jacobson, Phys. Rev. Lett. 50, 896 (1983).

[7] H.A. Atwater, J.A. West, P.M. Smith, M.J. Aziz, J.Y. Tsao, P.S. Peercy, and M.O. Thompson, Mat. Res. Soc. Symp. Proc. 157, 369 (1990).

[8] P.M. Smith, J.A. West, and M.J. Aziz, Mat. Res. Soc. Symp. Proc. 205, in press (1991).

[9] A.R. Yavari and B. Bochu, Phil. Mag. A 59, 697 (1989).

[10] B.P. Burton, A. Pasturel, J.E. Osborn, and W.C. Carter, these proceedings. 